\title{
A Sensitivity Analysis on the Spectral Signatures of Low-Backscattering Sea Areas in Sentinel-1 SAR Images
}

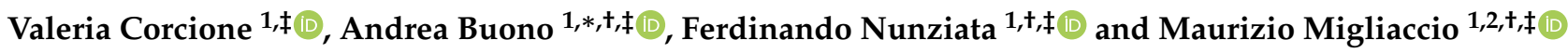 \\ 1 Dipartimento di Ingegneria, Università di Napoli Parthenope, Centro Direzionale isola C4, \\ 80143 Naples, Italy; valeria.corcione@uniparthenope.it (V.C.); ferdinando.nunziata@uniparthenope.it (F.N.); \\ maurizio.migliaccio@uniparthenope.it (M.M.) \\ 2 Istituto Nazionale di Geofisica e Vulcanologia, Via di Vigna Murata 605, 00143 Rome, Italy \\ * Correspondence: andrea.buono@uniparthenope.it \\ + Current address: Centro Direzionale isola C4, 80143 Napoli, Italy. \\ $\ddagger \quad$ These authors contributed equally to this work.
}

Citation: Corcione, V.; Buono, A.;

Nunziata, F.; Migliaccio, M. A

Sensitivity Analysis on the Spectral Signatures of Low-Backscattering Sea Areas in Sentinel-1 SAR Images.

Remote Sens. 2021, 13, 1183.

https://doi.org/10.3390/rs13061183

Academic Editor: Martin Gade

Received: 29 December 2020

Accepted: 16 March 2021

Published: 19 March 2021

Publisher's Note: MDPI stays neutral with regard to jurisdictional claims in published maps and institutional affiliations.

Copyright: (c) 2021 by the authors. Licensee MDPI, Basel, Switzerland. This article is an open access article distributed under the terms and conditions of the Creative Commons Attribution (CC BY) license (https:/ / creativecommons.org/licenses/by/ $4.0 /)$.
Abstract: Satellite synthetic aperture radar (SAR) is a unique tool to collect measurements over sea surface but the physical interpretation of such data is not always straightforward. Among the different sea targets of interest, low-backscattering areas are often associated to marine oil pollution even if several physical phenomena may also result in low-backscattering patches at sea. In this study, the effects of low-backscattering areas of anthropogenic and natural origin on the azimuth autocorrelation function (AACF) are analyzed using VV-polarized SAR measurements. Two objective metrics are introduced to quantify the deviation of the AACF evaluated over low-backscattering areas with reference to slick-free sea surface. Experiments, undertaken on six Sentinel-1 SAR scenes, collected in Interferometric Wide Swath $\mathrm{VV}+\mathrm{VH}$ imaging mode over large low-backscattering areas of different origin under low-to-moderate wind conditions (speed $\leq 7 \mathrm{~m} / \mathrm{s}$ ), spanning a wide range of incidence angles (from about $30^{\circ}$ up to $46^{\circ}$ ), demonstrated that the AACF evaluated within low-backscattering sea areas remarkably deviates from the slick-free sea surface one and the largest deviation is observed over oil slicks.

Keywords: SAR; sentinel-1; low-backscattering areas; azimuth autocorrelation function

\section{Introduction}

Continuous and effective monitoring of the oceans is of paramount importance to improving global marine awareness and the understanding of ocean dynamics, including man-made target surveillance, pollution monitoring, and the impact on climate change [1]. Satellite Earth observation represents a valuable tool that provides extensive data collection over the oceans. An important sensor for ocean observation is the synthetic aperture radar (SAR), an active, band-limited, and coherent microwave imaging sensor that provides day and night imagery in almost all-weather conditions [2]. The exploitation of SAR imagery for marine and maritime applications is now well-established [3-6] and has been further boosted when the European Space Agency (ESA) started providing Sentinel-1 (S1) SAR satellite measurements free of charge in 2014 [7-10].

The general physical modeling that rules sea surface scattering in SAR imagery accounts for both the sea surface roughness, i.e., the sea surface spectrum, and the sea permittivity. In the context of marine pollution, low-backscattering areas, whose normalized radar cross section (NRCS) is lower than the surrounding sea one, represent a broad class of targets of interest since they can be often associated to natural or anthropogenic oil slicks. Even though low-backscattering sea areas may have different origins, e.g., organic films, low-wind areas, etc., most analyses on low-backscattering areas have been focused on oil spill detection and classification [11-17], with important advancements that have 
been recently accomplished by means of proper physical processing of polarimetric SAR data [18-21].

Low-backscattering areas are due to the damping of the capillary and small gravity sea waves generated by the local wind and responsible for the measured NRCS, i.e., the centimetric Bragg resonant waves, which result in a reduced sea surface roughness. Accordingly, most of the incident energy is scattered in the specular direction rather than toward the SAR antenna. This is why low-backscattering sea areas appear as dark patches in graytones intensity SAR images [22,23]. However, note that the NRCS values depend on incident wavelength, polarization, and incidence angle [22]. In this paper, a new approach is proposed to exploit VV-polarized, i.e., vertical transmit/vertical receive, S1 SAR data, collected in interferometric wide (IW) swath mode, to characterize different kinds of large low-backscattering sea areas of a known origin. The approach, based on the autocorrelation function evaluated along the azimuth (AACF), relies on the spectral information inherently carried on by SAR measurements and on the SAR peculiar imaging mechanism along the azimuth [24]. The experimental analysis, performed under low-tomoderate wind regime, i.e., wind speed $\leq 7 \mathrm{~m} / \mathrm{s}$, and in a broad range of incidence angles (approximately $30^{\circ}-46^{\circ}$ ), considers slick-free sea surface as the reference scenario. The deviation of the AACF evaluated over low-backscattering areas of both an anthropogenic and natural origin is quantified using two objective metrics, namely the Euclidean distance and the percentage relative difference. Furthermore, the AACF analysis is compared to the conventional contrast, i.e., the slick-free sea surface to low-backscattering area NRCS ratio, and the effect of incidence angle is also discussed. The experimental results show a pronounced sensitivity of the AACF to low-backscattering sea areas. According to the metrics, the oil AACF differs from the slick-free sea surface AACF and from other natural low-backscattering area AACFs. In addition, the Euclidean distance, if compared to the NRCS contrast, is less dependent on the incidence angle.

The remainder of this paper is organized as follows: The theoretical background is presented in Section 2, while the dataset is described in Section 3. The experiments are presented in Section 4, where the results are discussed, while conclusions are drawn in Section 5 .

\section{Theoretical Background}

\subsection{Physical Rationale}

The SAR estimates the sea reflectivity by means of two different scanning mechanisms. The range or across-track direction imaging is done at the speed of light, therefore insensitive to temporal changes of the sea, while the azimuth or along-track direction imaging is done at satellite velocity, therefore sensitive to temporal changes of the sea [24-26]. The microwave signal scattered by the sea, under low-to-moderate sea state conditions and in a broad range of incidence angles, can be well described by two-scale scattering models [27]. The class of two-scale scattering models assumes that the full-range sea surface roughness spectrum is artificially split into two parts: The larger-scale roughness, mainly associated to longer surface waves, and the smaller-scale roughness, mainly associated to shorter surface waves, which are responsible for the Kirchhoff and Bragg scattering, respectively [28]. The choice of the $K_{\text {lim }}$, i.e., the wavenumber that splits the sea surface spectrum, has been addressed in several papers and is summarized in $[28,29]$ and references therein.

These two contributions are not independent since, for instance, the non-linear interaction between the capillary (shorter) and gravity (longer) waves is the physical mechanism that underpins the energy transfer from the wind to the waves [30]. However, to achieve a good compromise between accuracy, practical implementation, and interpretation, these two contributions are added incoherently [27]:

$$
\sigma_{p q}^{0}=\sigma_{p q, 0}^{0}+\sigma_{p q, 1^{\prime}}^{0}
$$


where $\sigma_{p q}^{0}, \sigma_{p q, 0}^{0}$, and $\sigma_{p q, 1}^{0}$ are the total NRCS, the zeroth-order Kirchhoff scattering contribution, and the first-order tilted-Bragg scattering contribution, respectively [28]. In (1), the subscripts " $q$ " and " $p$ " stand for transmitted and received polarization, respectively. In the S1 IW SAR imaging mode, the incidence angle is such that the main term contributing to $\sigma_{p q}^{0}$ is the first-order Bragg scattering [28]. The latter, which can be considered as an average of the untilted Bragg scattering over the larger-scale ripple by the long-waves structure, broadens the spectrum of the Bragg resonant waves [28]. Hence, according to the tilted-Bragg model, the microwave signal backscattered to the radar antenna depends directly on the small-scale ripple (through the small-scale sea surface roughness spectrum) and, indirectly, on the longer-wave part of the spectrum through the probability density function of the slopes [28,31,32].

The presence of a surfactant over the sea surface affects both the NRCS and SAR image spectrum [33]. In fact, it modifies the full-range sea surface spectrum [28], i.e., both the short- and the longer-wave part of the sea surface spectrum are affected by the surfactant. The visco-elastic properties of the surfactant have a direct impact on the small-scale part of the spectrum through a damping coefficient that, in the case of monomolecular surface films, can be modeled by the Marangoni damping coefficient [34]. In this case, as expected, the Marangoni damping mainly affects the small-scale part of the sea surface spectrum. The surfactant, reducing the sea surface roughness, also affects the energy transfer from the wind to the sea waves. The latter is typically modeled by the friction velocity, with this phenomenon that is well described by a reduced friction velocity over the surfactant. A reduced friction velocity has a direct implication on the long-wave part of the sea surface spectrum by modifying the peak wavenumber and the significant slope [28].

The above-described physical rationale does not explicitly take into account sea dynamic processes that are of paramount importance when dealing with SAR imaging of sea surface. The effect of the sea dynamics on SAR images is known as the velocity bunching phenomenon $[35,36]$ that is related to the azimuth channel. This imaging is affected by the scene coherence time which, being shorter than the SAR integration time, makes the SAR imaging act as a non ideal filter along the azimuth direction [25,37].

These dynamics processes, which make SAR images appear blurred in the azimuth direction [36], are such that the "optimal" focusing depends on the SAR image patch [36] and it can narrow /broaden according to the sea state [24]. Although all this matter was first seen as a limit in SAR imaging of the oceans [24], it was later considered as a geophysical information to be potentially exploited [36]. This phenomenon is known as azimuth cut-off [38]. In filter theory words, one can say that the azimuth cut-off is a measure of the actual SAR azimuth spatial resolution [24]. In [35] the azimuth cut-off is explained as being due to two main contributions. The radial velocity to the radar of the single moving water particle generates a Doppler shift with respect to a stationary scene and the Doppler shifts of the elementary scatterers in the SAR resolution cell are not all identical, i.e., the single elementary Doppler shift is different from the mean Doppler shift of the SAR resolution cell, producing a "velocity spread" that smears the image of the resolution cell [24,35].

The physical model detailed in [35] shows that the quasi-linear approximation of the SAR image power spectrum $P_{q l}(K)$ is modeled as a perturbation of the linear SAR image power spectrum $P_{l}(\boldsymbol{K})$ modeled in accordance to the linear imaging theory. Hence, the quasi-linear SAR image power spectrum $P_{q l}(\boldsymbol{K})$ can be expressed in function of the linear SAR image power spectrum $P_{l}(\boldsymbol{K})$ as follows [33,35]:

$$
P_{q l}(\boldsymbol{K})=\exp \left(-K_{x}^{2} \tilde{\zeta}^{2}\right) P_{l}(\boldsymbol{K}),
$$

where the subscript $x$ refers to the azimuth direction and $\xi^{2}$ is the total variance of the azimuthal displacements within the SAR integration time.

Equation (2) means that the SAR image spectrum is not able to represent the waves whose wavelength is less than the so-called azimuth cut-off wavelength $\lambda_{c}$, which can be interpreted as a measure of the low-pass filtering, in the wavenumber domain, witnessed by 
the exponential term. In (2), $\xi$ and consequently $\lambda_{\mathrm{c}}$ are related to the sea surface spectrum as follows [38]:

$$
\lambda_{\mathrm{c}}(\theta, \phi)=\pi \frac{R(\theta)}{V} \sqrt{\int_{0}^{\infty} \Omega^{2} S(K) F(\boldsymbol{K}, \theta, \phi) \mathrm{d} \boldsymbol{K},}
$$

where $\theta$ is the incidence angle, $\phi$ is the wind mean direction relative to the range axis [38], $R / V$ is the ratio between the slant-range distance and the velocity of SAR platform, $S(\cdot)$ is the omni-directional sea spectrum and $F(\cdot)$ is the directional sea spectrum. This reasoning can be exploited over slick-free sea surface, by means of a tailored semi-empirical model and proper estimation procedure, to determine the wind speed [33,39].

\subsection{Methodology}

In this study, the large degree of heterogeneity resulting from the low-backscattering sea class prevents the use of a compact parameter for the spectral analysis as in the case of wind speed estimation, that relies on $\lambda_{c}$. Hence, here the analysis is focused on the AACF that is obtained as the inverse Fourier transform of the power spectral density (PSD) following a guideline similar to the one proposed in [39] to estimate $\lambda_{\mathrm{c}}$. We expect a sensitivity of the AACF to the low-backscattering areas. In fact, the theoretical modeling here outlined shows that the surfactant affects the NRCS and the SAR image spectrum. The relationship between the slick-covered sea surface spectrum and the SAR image spectrum is generally complex and to some extent poorly known. Since the friction velocity reduction affects the dynamic process, it is expected to have an additional impact on the azimuthal SAR spectrum. Hence, we expect that the AACF evaluated over low-backscattering areas exhibits deviation from the reference slick-free sea surface one.

In detail, the methodology proposed to process S1 SAR imagery using the AACF can be summarized as follows, see also the flowchart of Figure 1:

- The VV-polarized uncalibrated intensity image of the SAR scene is divided into nonoverlapped square boxes whose size is $n=128$ pixels. This value is set according to the $\mathrm{S} 1$ pixel spacing, i.e., $10 \mathrm{~m}$, in order to ensure both a satisfactory degree of homogeneity and a consistent number of samples for a reliable AACF estimation;

- For each box, the 2-D PSD is evaluated as the square modulus of the Fourier transform of the VV-polarized uncalibrated intensity image;

- The 1-D azimuth PSD, PSD $_{x}$, is evaluated by averaging the PSD along the range direction;

- The AACF is obtained by applying the inverse Fourier transform:

$$
\mathrm{AACF}=\frac{1}{n^{2}} \operatorname{IFFT}\left(\mathrm{PSD}_{\mathrm{x}}\right),
$$

where PSD $=\operatorname{FFT}(X)$, with $X$ is the VV-polarized uncalibrated intensity SAR image;

- A smoothing $7 \times 1$ median filter is applied to the modulus of the azimuth autocorrelation function $(\mathrm{AACF})$ in order to remove the 0 -lag contribution. 


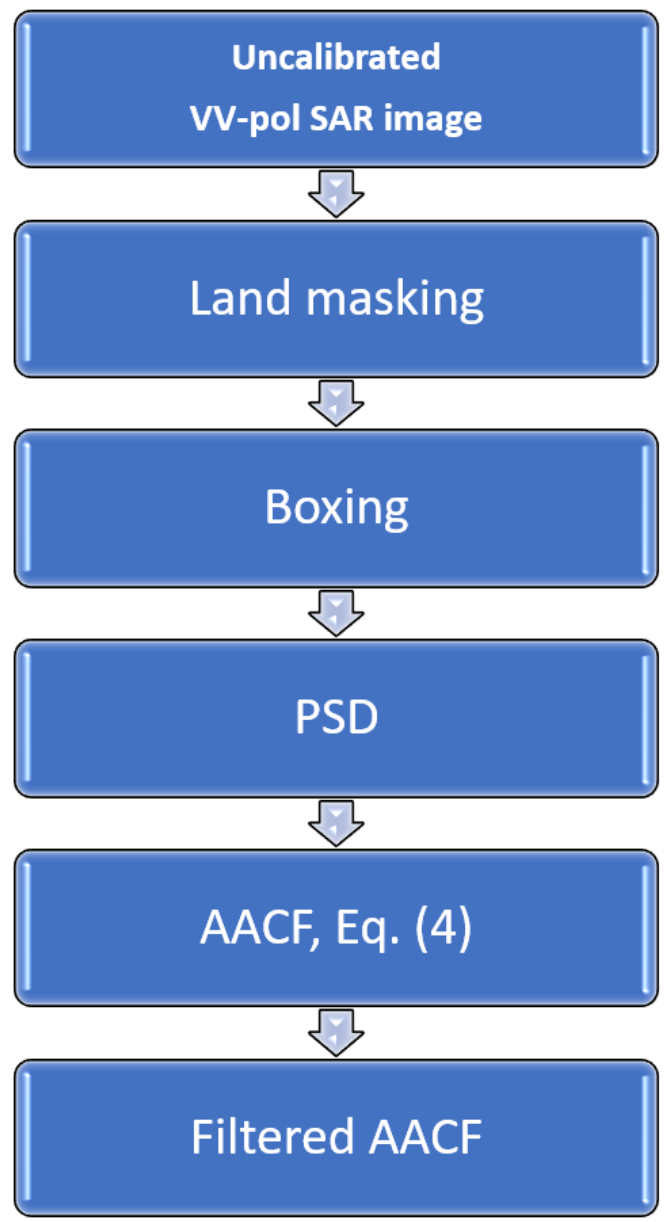

Figure 1. Flowchart of the AACF estimation procedure.

\section{Dataset}

The Copernicus Earth observation S1 mission consists of two polar-orbiting satellites equipped with a C-band $(5.405 \mathrm{GHz}) \mathrm{SAR}$. The latter supports dual-polarimetric imaging modes through a single switchable linear polarization transmission (horizontal- $\mathrm{H}$, or vertical-V) while receiving coherently in a linear orthogonal polarization basis H-V. S1 operates in four different acquisition modes: Stripmap, extra-wide swath, wave mode, and IW mode. The IW mode is composed by three sub-swaths obtaing using the terrain observation with progressive scans SAR (TOPSAR) imaging technique. The TOPSAR technique, together with the electronic steering of the beam, result in a high-quality image characterized by no scalloping and homogeneity throughout the swath.

In this study, a SAR dataset consisting of $6 \mathrm{C}$-band $\mathrm{S} 1 \mathrm{VV}+\mathrm{VH}$ ground range detected images collected in IW mode is considered that allows combining a large swath width $(250 \mathrm{~km})$ with a moderate geometric resolution $(5 \mathrm{~m}$ by $20 \mathrm{~m})$. The swath coverage is around $250 \mathrm{~km}$, the pixel spacing is $10 \mathrm{~m}$, and the incidence angle $\theta$ spans from about $30^{\circ}$ up to $46^{\circ}$. Although both co- and cross-polarized channels are available, in this study only co-polarized S1 imagery is used since the cross-polarized channel is significantly affected by noise due to the smaller signal-to-noise ratio (SNR) and, in addition, it exhibits a low sensitivity to low-backscattering areas. All the images are characterized by low-to-moderate wind conditions, i.e., wind speed between $2 \mathrm{~m} / \mathrm{s}$ and $7 \mathrm{~m} / \mathrm{s}$, therefore representing an optimal range for studying biogenic surfactants and oil slicks as suggested in [40]. The wind speeds are provided by space/time co-located ancillary European Centre for Mediumrange Weather Forecasting (ECMWF) information.

The S1 SAR images include low-backscattering areas of a known origin [41-47] related to both verified oil slicks and other natural phenomena, see Table 1 . The corresponding VV-polarized NRCS images are shown in Figure 2, where a graytones dB scale is used. 
The regions of interest (ROIs) considered for the experimental analysis are highlighted with dashed boxes that refer to slick-free sea surface (blue and red), oil slick (green), and look-alike (orange).

The image shown in Figure 2a is acquired on 10 August 2017, off the southern coast of Kuwait in the Persian Gulf, where a certified oil spill is present [41]. The oil spill is likely due to an accidental collision between the pipeline laying vessel "DLB 1600" and an old pipeline on the seafloor. A conservative estimation of the oil-covered area is $131 \mathrm{~km}^{2}$. Another low-backscattering area, due to very low wind conditions $(<3 \mathrm{~m} / \mathrm{s})$, is also present. The incidence angle relevant to both low-backscattering areas is equal to $32^{\circ}$.

The image shown in Figure $2 \mathrm{~b}$ was acquired on 8 October 2018, over the northern part of the Tyrrhenian Sea between Corsica and Tuscany coasts. A 20-km-long oil spill due to an accidental collision between two cargo ships the day before is present [42,43]. A large low-backscattering area due to very calm sea state is also present. The incidence angles over the two ROIs are $33^{\circ}$ and $41^{\circ}$, respectively.

The image shown in Figure 2c is acquired on 8 March 2017, off the coast of Fujairah in the western coast of the United Arab Emirates (Persian Gulf). Multiple oil slicks due to a seafloor leakage from the jack-up drill rig "Pasargad 100" are present [44]. The polluted sea area is conservatively estimated to be about $334 \mathrm{~km}^{2}$. The incidence angle over the ROIs is $35^{\circ}$.

The image shown in Figure 2d was acquired on 11 March 2017, over the same area of Figure 2c, during the same oil spill event. Another low-backscattering area due to very low wind conditions $(<3 \mathrm{~m} / \mathrm{s})$ is also visible [44]. The incidence angle over the ROIs are $35^{\circ}$ and $40^{\circ}$, respectively.

The image shown in Figure 2e was acquired on 20 July 2019, around the Gotland island in the Baltic Sea between Sweden and Latvia. A diffuse low-backscattering area due to swirling green algae blooms covers most of the observed sea surface [45]. The incidence angle measured at mid-range is equal to $38^{\circ}$.

The image shown in Figure $2 \mathrm{f}$ was acquired on 1 April 2018, over the Balikpapan Bay on the eastern coast of Indonesia. An oil spill due to a 25-m-underwater oil pipeline damaged the day before is present. The oil-affected area was estimated to be about $130 \mathrm{~km}^{2}[46,47]$. The incidence angle over the oil slick is $43^{\circ}$. 


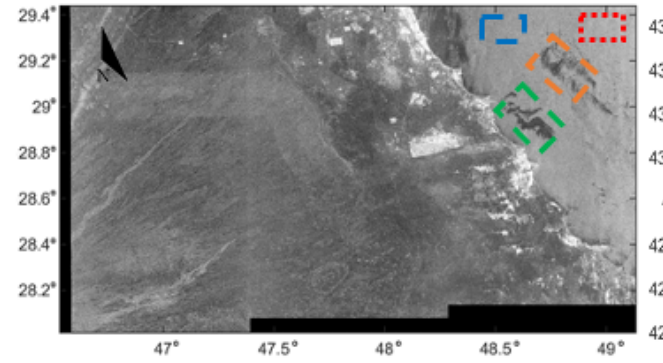

(a)

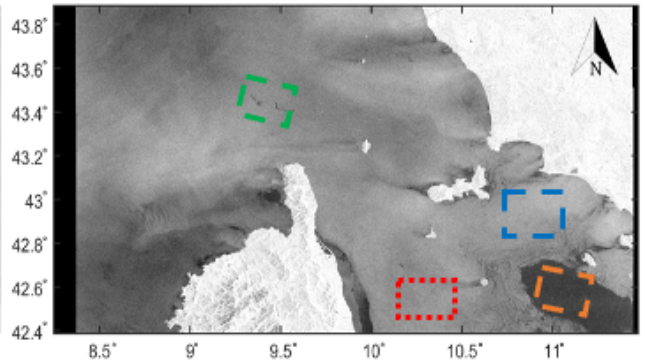

(b)

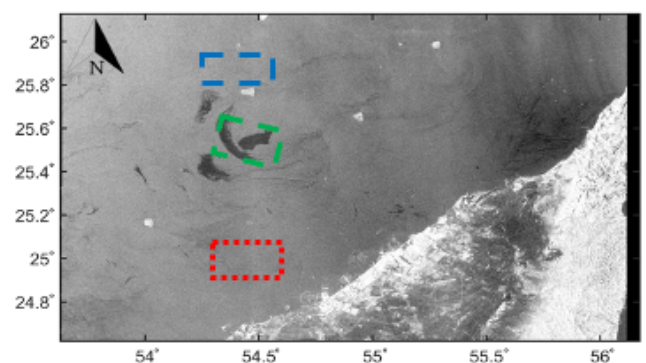

(c)

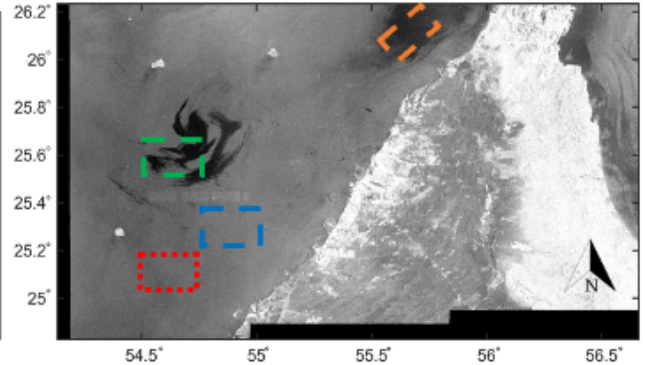

(d)

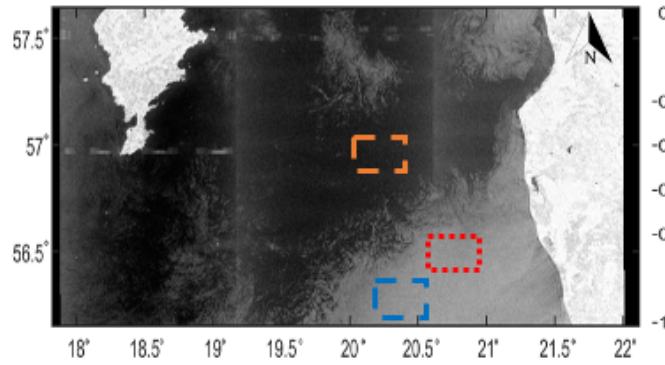

(e)

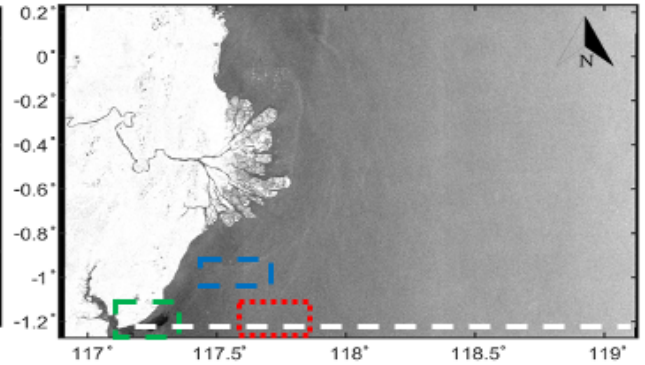

(f)
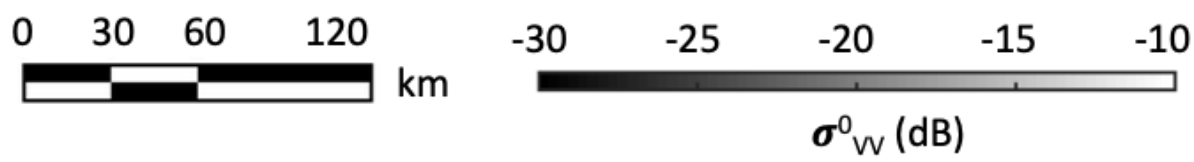

Figure 2. S1 synthetic aperture radar (SAR) dataset: VV-polarized normalized radar cross section (NRCS) graytones images (in $\mathrm{dB}$ scale) that include low-backscattering areas. (a-f) refer to the SAR scenes labeled as data ID 1-6 in Table 1. The regions of interest (ROIs) that refer to slick-free sea surface, oil slick, and look-alike are highlighted with blue-, green-, and orange-dashed boxes, respectively. An extra slick-free sea surface ROI, highlighted with a dashed red box, is also considered to analyze the intrinsic sea surface variability. 
Table 1. Main features of the S1 SAR dataset.

\begin{tabular}{ccccccc}
\hline $\begin{array}{c}\text { Data } \\
\text { ID }\end{array}$ & $\begin{array}{c}\text { Acquisition } \\
\text { Date }\end{array}$ & Figure & $\begin{array}{c}\text { ROIs Wind } \\
\text { Speed }(\mathbf{m} / \mathbf{s})\end{array}$ & ROIs & Reference \\
\hline 1 & $10 / 8 / 2017$ & Figure 2a & $2-3$ & Slick-free sea surface, oil slick, look-alike & {$[41]$} \\
2 & $8 / 10 / 2018$ & Figure 2b & $<3 \& 4-6$ & Slick-free sea surface, oil slick, look-alike & {$[42,43]$} \\
3 & $8 / 3 / 2017$ & Figure 2c & 5 & Slick-free sea surface, oil slick & {$[44]$} & {$[44]$} \\
4 & $11 / 3 / 2017$ & Figure 2d & $<3$ & Slick-free sea surface, oil slick, look-alike & {$[45]$} \\
5 & $20 / 7 / 2019$ & Figure 2e & $<3$ & Slick-free sea surface, look-alike & {$[46,47]$} \\
6 & $1 / 4 / 2018$ & Figure 2f & $6-7$ & Slick-free sea surface, oil slick & \\
\hline
\end{tabular}

\section{Experiments}

In this section, the sensitivity of the AACF to the low-backscattering areas highlighted in Figure 2 is analyzed.

\subsection{Experimental Settings}

The AACF over each ROI is estimated by averaging at least 10 AACFs evaluated according to the methodology described in Section 2. To allow a fair intercomparison of the estimated AACFs, they are normalized to their maximum value and the pedestal is set to zero, as it will be shown in Section 4.2, where the color coding is in accordance to the ROIs of Figure 2. In all the subsequent experiments, reference is made to slick-free sea surface ROIs (that will be labeled in blue as "Sea ref") at almost the same incidence angle of the low-backscattering areas, see dashed blue boxes in Figure 2. In each SAR image, an extra slick-free sea surface ROI (that will be labeled in red as "Sea"), see the dashed red boxes of Figure 2, is also selected to analyze the intrinsic sea surface variability. In the following, we refer to the slick-free sea surface $A A C F$ s as $A A C F_{s}$ and to the AACFs estimated within the low-backscattering areas as $A A C F_{l b}$. In all subsequent $S A R$ image analysis, the average contrast $\Delta$, i.e., the difference between the slick-free sea surface and the low-backscattering area NRCS values in $\mathrm{dB}$ scale, is evaluated as follows:

$$
\Delta(\mathrm{dB})=10 \log _{10}\left(\sigma_{\mathrm{VV}}^{0}\right)_{\mathrm{s}}-10 \log _{10}\left(\sigma_{\mathrm{VV}}^{0}\right)_{\mathrm{lb}}
$$

where the subscripts " $\mathrm{s}$ " and "lb" stand for slick-free sea surface and low-backscattering area, respectively.

To quantify the deviation of the $\mathrm{AACF}_{\mathrm{lb}}$ with respect to the $\mathrm{AACF}_{\mathrm{S}}$, the Euclidean distance, $d_{\mathrm{E}}$, and the percentage relative difference, $D_{\text {rel }}$, are introduced. The Euclidean distance is defined as:

$$
d_{\mathrm{E}}=\sqrt{\sum_{i=1}^{m}\left(l b_{i}-s_{i}\right)^{2}},
$$

where $l b$ and $s$ refer to $A A C F$ lb and $A A C F$, respectively, while $i$ is the index that refers to the AACF samples of the selected low-backscattering and slick-free sea surface ROIs, each consisting of $m=1000$ pixels. The percentage relative difference is defined as:

$$
D_{\text {rel }}(\%)=\frac{\left(d_{\mathrm{E}}\right)_{\mathrm{lb}}-\left(d_{\mathrm{E}}\right)_{\mathrm{s}}}{\left(d_{\mathrm{E}}\right)_{\mathrm{lb}}} \times 100,
$$

where $\left(d_{\mathrm{E}}\right)_{\mathrm{lb}}$ is the Euclidean distance between the low-backscattering ROI and the reference sea surface one, while $\left(d_{\mathrm{E}}\right)_{\mathrm{S}}$ is the Euclidean distance between the two slick-free surface ROIs. This metric, as it is defined, is thought to analyze the deviation of the AACF estimated over low-backscattering areas from the corresponding slick-free sea surface reference one in order to filter out the intrinsic sea surface variability, i.e., induced by NRCS variability with respect to the azimuth angle (wind direction relative to the radar's azimuth look direction). All the above-mentioned quantitative parameters are listed in Table 2. Before proceeding further with the AACF analysis, since we are dealing with lowbackscattering areas, a discussion on the effects of additive noise onto the backscattered 
signal is due. When dealing with S1 SAR data, the worst case nominal noise equivalent sigma zero (NESZ) is $-22 \mathrm{~dB}$. Hence, a received signal whose intensity is lower than NESZ may be uninformative. To perform a more accurate analysis, the SNR is used. The latter is not evaluated using the provided (worst case) NESZ but is estimated from the data according to $[48,49]$ :

$$
\mathrm{NESZ}=\frac{\eta}{A^{2}}
$$

where $\eta$ and $A$ are the noise calibration parameter and the NRCS calibration factor, respectively, which are pixel-dependent parameters provided in the ESA annotated metadata through a look-up-table. Then, the SNR is evaluated as follows:

$$
\mathrm{SNR}(\mathrm{dB})=10 \log _{10}\left(\sigma_{\mathrm{VV}}^{0}\right)-10 \log _{10}(\mathrm{NESZ}) \text {. }
$$

\subsection{Discussion}

The SNR images evaluated over the whole SAR dataset are shown, in dB scale, in Figure 3, where land is masked in white. The average SNR values evaluated over the ROIs highlighted in Figure 2 are listed in Table 2.

Considering the reference slick-free sea surface ROIs (dashed blue boxes), the average SNR is always larger than about $7 \mathrm{~dB}$, witnessing that the signal scattered off slick-free sea surface lies well above the NESZ along the whole SAR dataset. Considering the lowbackscattering sea areas, i.e., both oil slicks and look-alikes, the average SNR estimated over the corresponding ROIs significantly varies along the SAR dataset depending on the damping properties and incidence angle. Oil slicks (dashed green boxes), call for an average SNR that lies in the range of $1.5 \mathrm{~dB}-4.2 \mathrm{~dB}$, while the look-alikes (dashed orange boxes), result in general in lower SNR values falling in the range of $0.5 \mathrm{~dB}-2.8 \mathrm{~dB}$. Note that these values can be either lower or larger than the oil slick ones. Hence, for the purposes of the spectral analysis, it can be concluded that reference slick-free sea surface samples are noise-free and oil slick samples can be considered to have an average SNR large enough to not affect significantly the spectral analysis, while look-alike samples are partly contaminated by noise and, therefore, particular attention must be paid in their spectral analysis.

The first experiment refers to the SAR image ID 1 , see Table 1 . The two low-backscattering ROIs are characterized by a great $\Delta$ value, i.e., $>6 \mathrm{~dB}$. The corresponding AACFs evaluated over the four ROIs are plotted in Figure 4a. The qualitative analysis clearly shows that the two $A A C F_{s}$ are narrow, while the two $A A C F_{l b}$ are wider. Although both $A A C F_{l b}$ show a distinct behavior with respect to $\mathrm{AACF}_{\mathrm{S}}$, the oil-covered one shows the largest broadening due to the oil damping properties and the reduction of the energy transfer from wind to the sea waves. It can be also noted that both ROIs call for a large enough average SNR, i.e., $>1.5 \mathrm{~dB}$. The low-wind ROI, whose SNR exhibits large spatial variability, calls for the largest average SNR $(2.8 \mathrm{~dB})$. The low-backscattering ROIs are characterized by $d_{\mathrm{E}}$ values showing a significant deviation from the reference $\mathrm{AACF}_{\mathrm{S}}$, i.e., 3.31 and 1.80 for the oil-covered and the low-wind ROI, respectively. The intrinsic sea variability, measured by computing the Euclidean distance $d_{\mathrm{E}}$ between the "Sea ref" and "Sea" AACFs, is equal to 0.30, witnessing a good overlapping of the corresponding $\mathrm{AACF}_{\mathrm{s}}$. Note that a similar result is obtained by randomly changing the position of the two slick-free sea surface ROIs. In conclusion, the $d_{\mathrm{E}}$ values relevant to the low-wind and oil-covered ROIs are about 6 and 11 times greater than 0.30 , respectively. The $D_{\text {rel }}$ values are $90.9 \%$ and $83.3 \%$ over the oil-covered and low-wind ROIs, respectively. 
Table 2. Experimental results relevant to the ROIs selected from the SAR dataset shown in Figure 2.

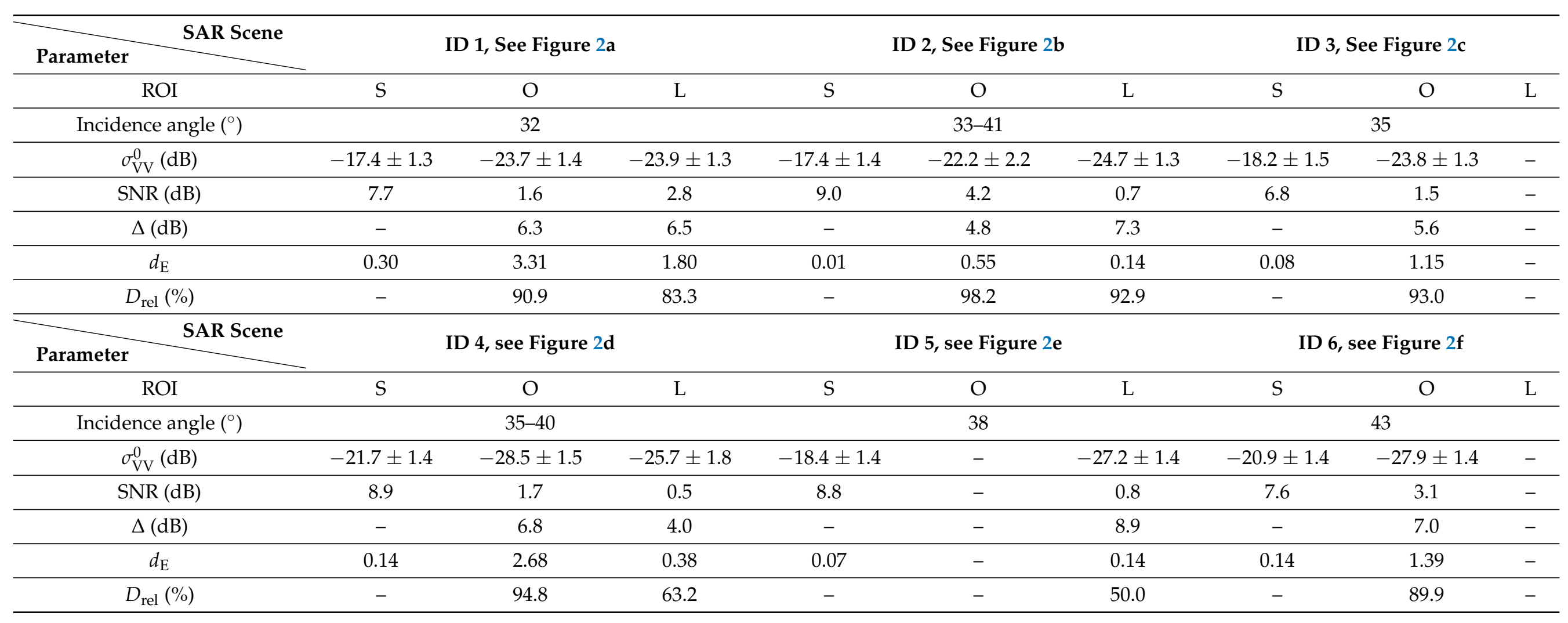




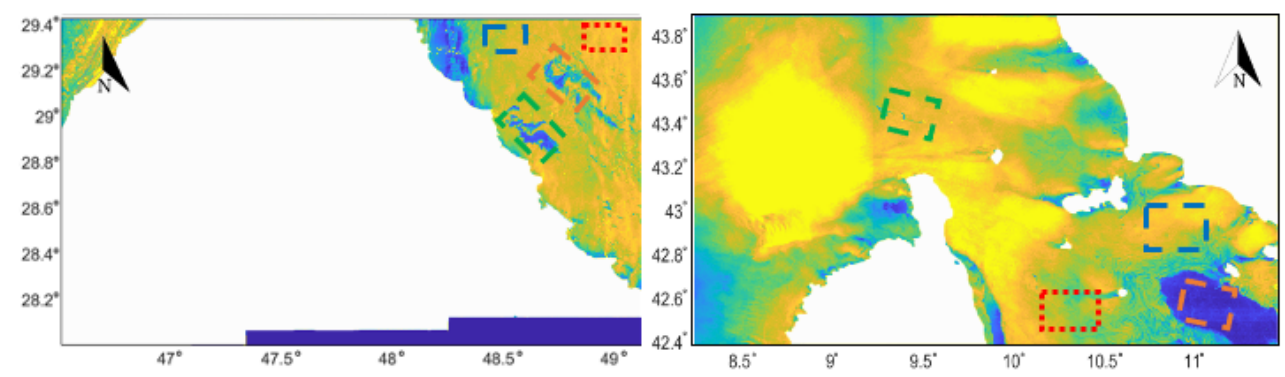

(a)

(b)

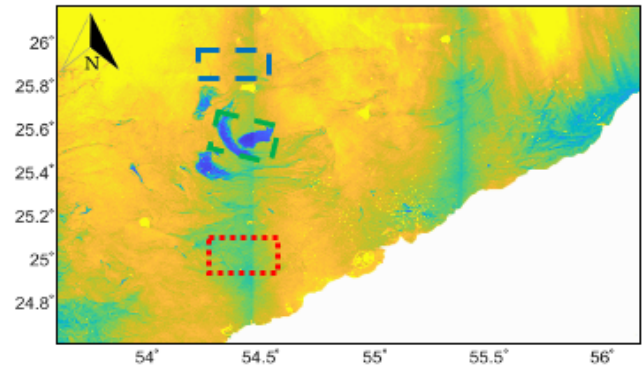

(c)

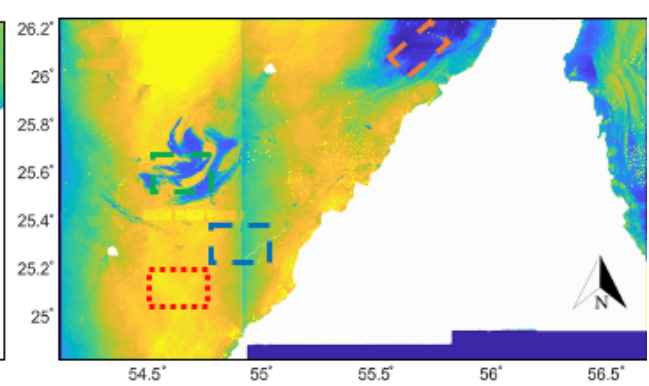

(d)

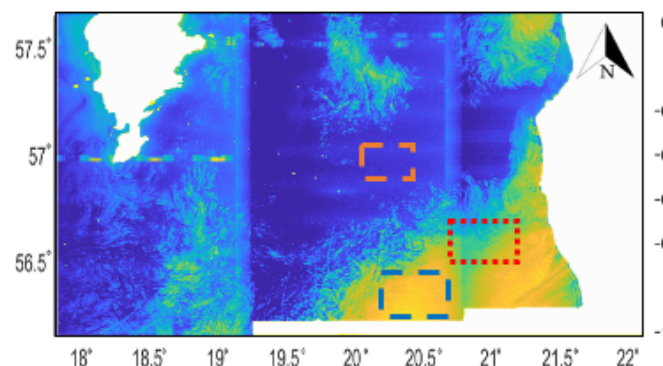

(e)

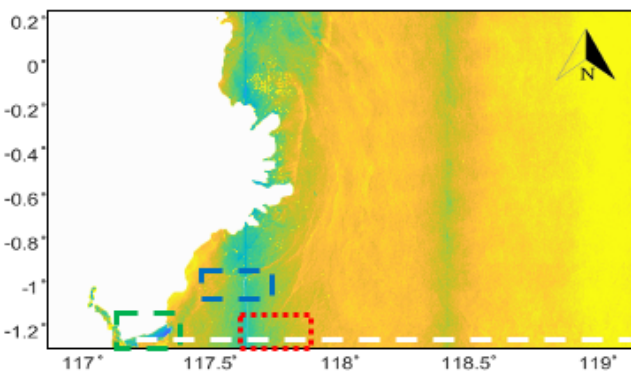

(f)
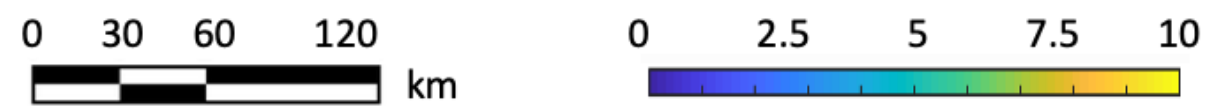

SNR (dB)

Figure 3. SNR images, in dB scale, evaluated over the SAR dataset shown in Figure 2. (a-f) refer to the SAR scenes labeled as data ID 1-6 in Table 1. Land is masked in white. 


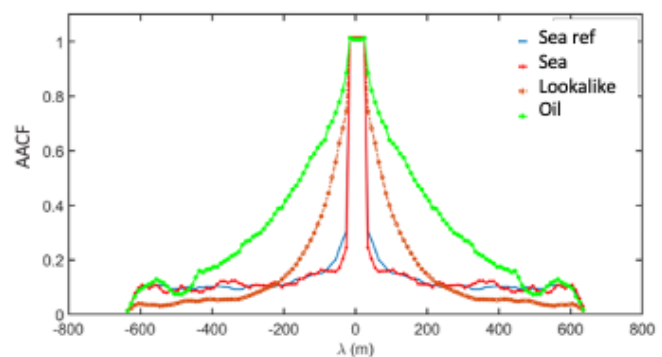

(a)

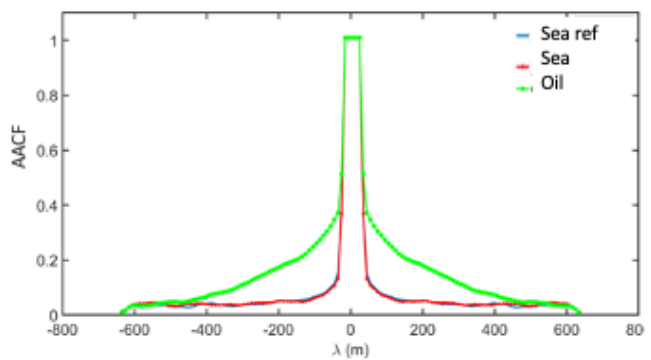

(c)

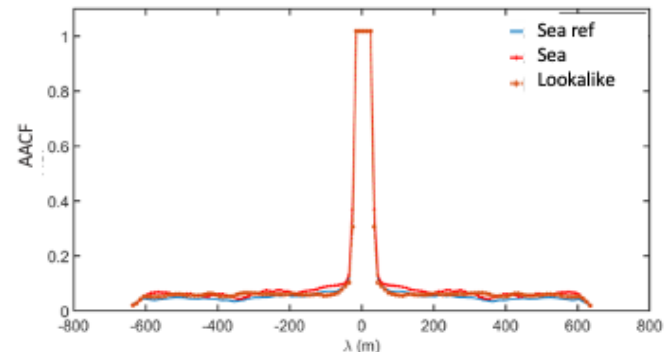

(e)

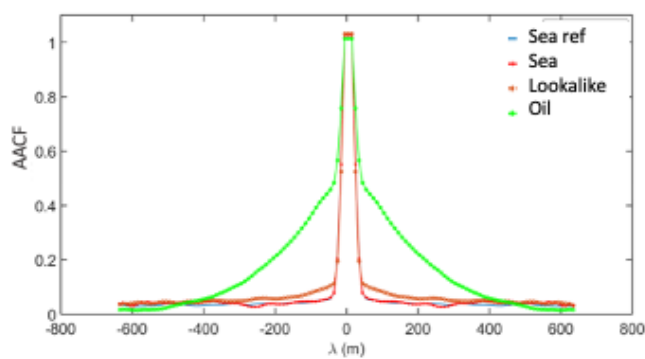

(b)

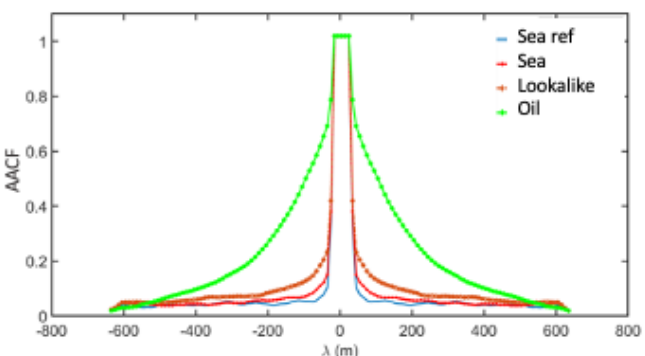

(d)

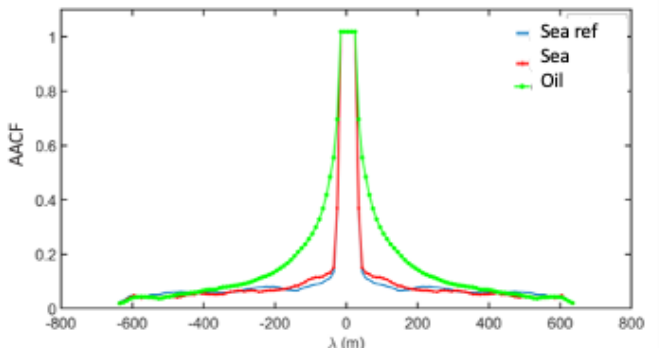

(f)

Figure 4. AACFs evaluated over the ROIs highlighted in Figure 2. (a-f) refer to the SAR scenes labeled as data ID 1-6 in Table 1 . Note that each AACF is normalized to its maximum value.

The second experiment refers to the SAR image ID 2, see Table 1. The two lowbackscattering ROIs show a great $\Delta$ value, i.e., about $5 \mathrm{~dB}$ and $7 \mathrm{~dB}$ for the oil-covered and low-wind ROI, respectively. The four AACFs are plotted in Figure $4 \mathrm{~b}$. The two $A A C F_{\mathrm{lb}}$ are wider than the two $\mathrm{AACF}_{\mathrm{S}}$, with the oil $\mathrm{AACF}_{\mathrm{lb}}$ resulting in the largest broadening. $d_{\mathrm{E}}$ is equal to 0.55 and 0.14 for the oil-covered and the low-wind ROI, respectively. This occurs even if the oil-covered ROI is characterized by an average SNR significantly larger than the low-wind $\mathrm{ROI}$ ( $4.2 \mathrm{~dB}$ versus $0.7 \mathrm{~dB}$, respectively), witnessing that the broadening is mainly due to the different spectral properties of the low-backscattering sea areas rather than to the additive noise. The intrinsic variability of the slick-free sea surface results in $d_{\mathrm{E}}=0.01$, witnessing a very small $\mathrm{AACF}_{\mathrm{S}}$ variability. The $d_{\mathrm{E}}$ values relevant to $\mathrm{AACF}_{\mathrm{lb}}$ are at least one order of magnitude larger than the ones relevant to $\mathrm{AACF}_{\mathrm{s}}$. The $D_{\text {rel }}$ values are $98.2 \%$ and $92.9 \%$ over the oil-covered and low-wind ROIs, respectively, showing a remarkable sensitivity of the AACF to the low-backscattering ROIs. In fact, $\mathrm{AACF}_{\mathrm{lb}}$ significantly deviates from $\mathrm{AACF}_{\mathrm{s}}$.

The third experiment refers to the SAR image ID 3 and 4, see Table 1 . The two scenes refer to the same oil slick observed in two different dates and under different sea state conditions. The SAR image of Figure $2 \mathrm{~d}$ also includes a low-wind ROI. Again, all the lowbackscattering ROIs are characterized by a great $\Delta$ value, $>4 \mathrm{~dB}$. The corresponding AACF 
evaluated over the considered ROIs are plotted in Figure $4 \mathrm{c}, \mathrm{d}$. The $\mathrm{AACF}_{\mathrm{s}}$ are narrower than the $\mathrm{AACF}_{\mathrm{lb}}$, with the oil $\mathrm{AACF}_{\mathrm{lb}}$ showing the largest broadening. Even in this case, the largest broadening of the oil-covered ROI cannot be attributed to the additive noise since the oil-covered ROI calls for an average SNR which is more than three times larger than the low-wind one ( $1.7 \mathrm{~dB}$ versus $0.5 \mathrm{~dB}$, respectively). For all the low-backscattering ROIs, $d_{\mathrm{E}}$ values are in the range of $0.38-2.68$, always larger than the intrinsic sea variability that results in $d_{\mathrm{E}}$ equal to 0.08 and 0.14 for SAR image ID 3 and 4 , respectively. The $d_{\mathrm{E}}$ values relevant to the oil and the low-wind $A A C F_{1 b}$ are more than one order of magnitude and just three times larger than the ones relevant to $\mathrm{AACF}_{\mathrm{S}}$, respectively. These results are consistent with the $D_{\text {rel }}$ values which are about $94 \%$ and $63 \%$ for the oil-covered and low-wind ROIs, respectively.

The fourth experiment refers to the SAR image ID 5, see Table 1 . The low-backscattering ROI shows a significantly great contrast, i.e., $\triangle$ about $9 \mathrm{~dB}$. The three AACFs are plotted in Figure $4 \mathrm{e}$, where it can be noted that $\mathrm{AACF}_{\mathrm{s}}$ and $\mathrm{AACF}_{\mathrm{lb}}$ overlap despite the remarkable contrast. Although it seems that the sensitivity of the AACF to this low-backscattering area is negligible, this is most likely due to the very low wind conditions that apply over the whole sea area. As a result, the longer-wave part of the spectrum does not change significantly from the slick-free sea surface to the low-backscattering ROI. It is interesting to note that $\mathrm{AACF}_{\mathrm{s}}$ and $\mathrm{AACF}$ lb call for almost the same width even if the average SNR of the low-backscattering $\mathrm{ROI}$ is more than 10 times lower than reference slick-free sea surface one ( $0.8 \mathrm{~dB}$ versus $8.8 \mathrm{~dB}$, respectively), witnessing that additive noise is not the factor driving the $\mathrm{AACF}$ broadening. However, although $\mathrm{AACF}_{\mathrm{s}}$ and $\mathrm{AACF}_{\mathrm{lb}}$ appear overlapped, they result in $d_{\mathrm{E}}=0.14$, i.e., twice the intrinsic sea variability $\left(d_{\mathrm{E}}=0.07\right)$. The corresponding $D_{\text {rel }}$ value is $50.0 \%$ that, even though it is the smallest value among the whole SAR dataset, still represents a remarkable deviation.

The fifth experiment refers to the SAR image ID 6, see Table 1. Even in this case, the oil-covered ROI shows a pronounced contrast, i.e., $\Delta=7 \mathrm{~dB}$. The three AACFs are plotted in Figure 4f. The oil $\mathrm{AACF}_{\mathrm{lb}}$ clearly deviates from the $\mathrm{AACF}_{\mathrm{s}}$. This broadening results in $d_{\mathrm{E}}=1.39,10$ times larger than the intrinsic sea variability $\left(d_{\mathrm{E}}=0.14\right)$. In this case, the oil-covered ROI calls for $D_{\text {rel }}=89.9 \%$.

The experimental results suggest that the NRCS and the AACF call for a different sensitivity to the incidence angle. Hence, a deeper investigation is due. The behavior of $\Delta$ and $d_{\mathrm{E}}$ with respect to the incidence angle is analyzed with reference to the scene depicted in Figure $2 \mathrm{f}$. The two metrics are evaluated fixing the oil-covered ROI while moving the slick-free see surface ROI along the range transect to span the available range of incidence angles. As suggested in $[48,50,51]$, for the incidence angles of interest $\left(31^{\circ}-43^{\circ}\right)$, the slick-free sea surface NRCS variability is expected to be large, i.e., about $10 \mathrm{~dB}$, while the oil NRCS variability is expected to be much smaller. Hence, $\Delta$ is expected to vary significantly with $\theta$. The experimental results are depicted in Figure 5 , where a dB scale is used. One can note, as expected, that $\Delta$ significantly varies with the incidence angle. This variability is practically negligible when $d_{\mathrm{E}}$ is considered. 


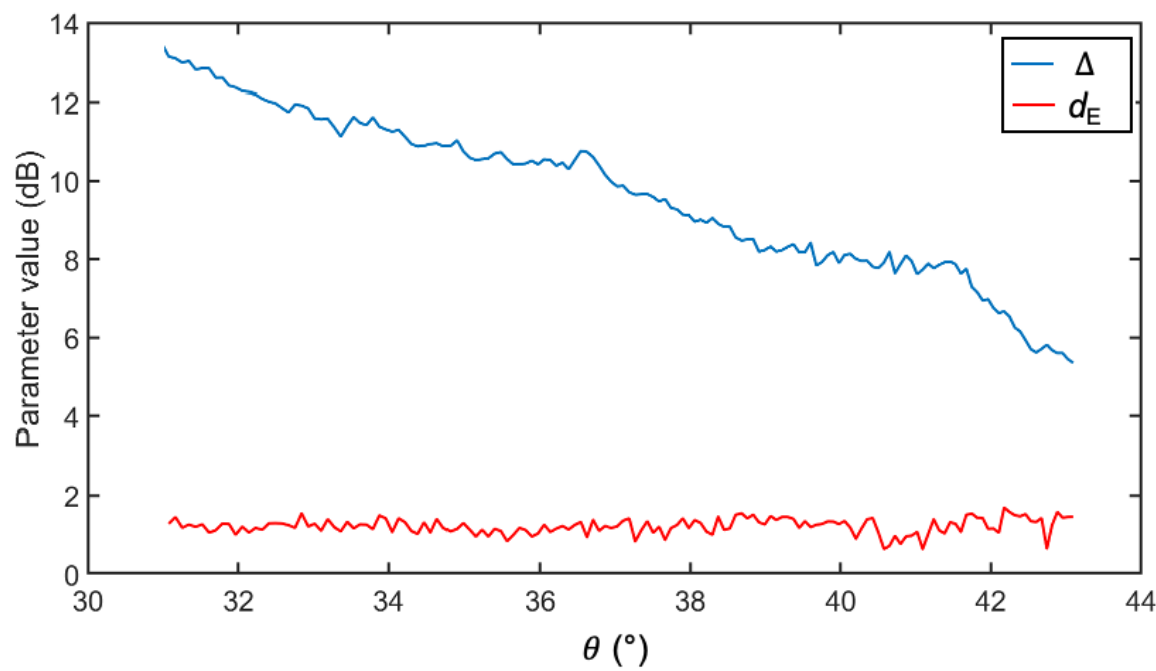

Figure 5. Behavior of the $\Delta$ and $d_{\mathrm{E}}$ parameters versus the incidence angle $\theta$ evaluated along with the dashed white range transect highlighted in Figure $2 \mathrm{f}$.

\section{Conclusions}

In this study, a spectral analysis of low-backscattering sea areas of both anthropogenic and natural origin was addressed. This is of great interest for the marine pollution community. The rationale of the proposed analysis relies on the capability of low-backscattering sea features, including oil slicks, algal blooms, and low-wind regions, to modify the full-range sea surface spectrum. Hence, the sensitivity of the SAR image autocorrelation function evaluated along the azimuth direction, namely AACF, was investigated.

The AACF estimated over low-backscattering areas was analyzed with reference to a slick-free sea surface AACF. The deviation of the low-backscattering AACF from the slickfree sea surface one was quantified according to two objective metrics, i.e., the Euclidean distance $d_{\mathrm{E}}$ and the percentage relative difference $D_{\text {rel }}$. A comparison was also made with respect to the intrinsic sea variability, i.e., the AACF deviation between two different slick-free sea surface areas.

Experiments, undertaken on 6 S1 VV-polarized SAR images, collected in IW dualpolarimetric imaging mode, where known oil slicks and low-backscattering sea areas due to natural phenomena are observed under low-to-moderate wind conditions $(2 \mathrm{~m} / \mathrm{s}-7 \mathrm{~m} / \mathrm{s})$ in a broad range of incidence angles $\left(\approx 30^{\circ}-46^{\circ}\right)$, showed that:

- $\quad$ The AACF is sensitive to different low-backscattering areas, with $d_{\mathrm{E}}$ and $D_{\text {rel }}$ values which are at least twice and 50\% larger, respectively, than the intrinsic sea surface variability;

- Among the low-backscattering sea areas, the oil slicks exhibit the largest AACF deviation with respect to the reference slick-free sea surface, with a maximum of $d_{\mathrm{E}}=3.31$ and $D_{\text {rel }}=98.2 \%$;

- $\quad$ The additive noise does not play a key role in broadening the AACF;

- $\quad$ The AACF is practically independent on the incidence angle while the backscattering contrast depends on it.

Author Contributions: Conceptualization, M.M.; methodology, F.N. and A.B.; software, V.C.; validation, V.C., A.B. and F.N.; formal analysis, V.C., A.B. and F.N.; data curation, V.C. and A.B.; writing-original draft preparation, A.B. and M.M.; writing-review and editing, F.N. and M.M; supervision, M.M. All authors have read and agreed to the published version of the manuscript.

Funding: This research was funded by the European Space Agency within the frame of ESA-MOST (Ministry of Science and Technology) Dragon 5 Cooperation (Monitoring harsh coastal environments and ocean surveillance using radar remote sensing sensors, Project ID 57979). 
Acknowledgments: The authors thank the European Space Agency for providing Sentinel-1 SAR data free of charge through the Copernicus Scientific Hub. This paper was written during the COVID-19 pandemic in Italy and is dedicated to all people working in the healthcare system.

Conflicts of Interest: The authors declare no conflict of interest.

$\begin{array}{ll}\text { Abbreviations } \\ \text { The following abbreviations are used in this manuscript: } \\ \text { AACF } & \text { azimuth autocorrelation function } \\ \text { ECMWF } & \text { European centre for medium-range weather forecasting } \\ \text { ESA } & \text { European space agency } \\ \text { FFT } & \text { fast Fourier transform } \\ \text { ID } & \text { identifier } \\ \text { IFFT } & \text { inverse fast Fourier transform } \\ \text { IW } & \text { interferometric wide } \\ \text { NESZ } & \text { noise equivalent sigma zero } \\ \text { NRCS } & \text { normalized radar cross section } \\ \text { PSD } & \text { power spectral density } \\ \text { ROI } & \text { region of interest } \\ \text { S1 } & \text { Sentinel-1 } \\ \text { SAR } & \text { synthetic aperture radar } \\ \text { SNR } & \text { signal-to-noise ratio } \\ \text { TOPSAR } & \text { terrain observation with progressive scans SAR } \\ \text { VH } & \text { vertical transmit horizontal receive } \\ \text { VV } & \text { vertical transmit vertical receive }\end{array}$

\section{References}

1. Visbeck, M. Ocean Science Research Is Key for a Sustainable Future. Nat. Commun. 2018, 9, 1-4. [CrossRef]

2. Li, X.; Guo, H.; Chen, K.-S.; Yang, X. Advances in SAR Remote Sensing of Oceans; Taylor \& Francis Group: Boca Raton, FL, USA; CRC Press: Boca Raton, FL, USA, 2018; 344p.

3. Alpers, W.; Holt, W.B.; Zeng, K. Oil Spill Detection by Imaging Radars: Challenges and Pitfalls. Remote Sens. Environ. 2017, 201, 133-147. [CrossRef]

4. Migliaccio, M.; Huang, L.; Buono, A. SAR Speckle Dependence on Ocean Surface Wind Field. IEEE Trans. Geosci. Remote Sens. 2019, 57, 5447-5455. [CrossRef]

5. Velotto, D.; Bentes, C.; Tings, B.; Lehner, S. First Comparison of Sentinel-1 and TerraSAR-X Data in the Framework of Maritime Targets Detection: South Italy Case. IEEE J. Ocean. Eng. 2016, 41, 993-1006. [CrossRef]

6. Shen, H.; Perrie, W.; He, Y.; Liu, G. Wind Speed Retrieval From VH Dual-Polarization RADARSAT-2 SAR Images. IEEE Trans. Geosci. Remote Sens. 2014, 52, 5820-5826. [CrossRef]

7. Hanowski, P. Copernicus Sentinel Data Access 2018 Annual Report; COPE-SERCO-RP-19-0389; Frascati, Italy, 2019.

8. Vachon, P.W.; Wolfe, J. GMES Sentinel-1 Analysis of Marine Applications Potential (AMAP); Defence Research and Development: Ottawa, ON, Canada, 2008.

9. Malenovsky, Z.; Rott, H.; Cihlar, J.; Schaepman, M.E.; García-Santos, G.; Fernandes, R.; Berger, M. Sentinels for Science: Potential of Sentinel-1, -2, and -3 Missions for Scientific Observations of Ocean, Cryosphere, and Land. Remote Sens. Environ. 2012, 120, 91-101. [CrossRef]

10. Misra, A.; Balaji, R. Simple Approaches to Oil Spill Detection Using Sentinel Application Platform (SNAP)-Ocean Application Tools and Texture Analysis: A Comparative Study. J. Indian Soc. Remote Sens. 2017, 45, 1065-1075. [CrossRef]

11. Trivero, P.; Fiscella, B.; Gomez, F.; Pavese, P. SAR Detection and Characterization of Sea Surface Slicks. Int. J. Remote Sens. 1998, 19, 543-548. [CrossRef]

12. Bertacca, M.; Berizzi, F.; Mese, E.D. A FARIMA-Based Technique for Oil Slick and Low-Wind Areas Discrimination in Sea SAR Imagery. IEEE Trans. Geosci. Remote Sens. 2005, 43, 2484-2493. [CrossRef]

13. Topouzelis, K. Oil Spill Detection by SAR Images: Dark Formation Detection, Feature Extraction and Classification Algorithms. Sensors 2008, 8, 6642-6659. [CrossRef]

14. Nunziata, F.; Buono, A.; Migliaccio, M. COSMO-SkyMed Synthetic Aperture Radar Data to Observe the Deepwater Horizon Oil Spill. Sustainability 2018, 10, 3599. [CrossRef]

15. Lang, H.; Zhang, X.; Xi, Y.; Zhang, X.; Li, W. Dark-Spot Segmentation for Oil Spill Detection Based on Multifeature Fusion Classification in Single-pol Synthetic Aperture Radar Imagery. J. Appl. Remote Sens. 2017, 11, 015006. [CrossRef]

16. Song, H.; Huang, B.; Zhang, K. A Globally Statistical Active Contour Model for Segmentation of Oil Slick in SAR Imagery. IEEE J. Selected Topics Appl. Earth Obs. Remote Sens. 2013, 6, 2402-2409. [CrossRef] 
17. Girard-Ardhuin, F.; Mercier, G.; Collard, F.; Garello, R. Operational Oil-Slick Characterization by SAR Imagery and Synergistic Data. IEEE J. Ocean. Eng. 2005, 30, 487-495. [CrossRef]

18. Buono, A.; Nunziata, F.; de Macedo, C.R.; Velotto, D.; Migliaccio, M. A Sensitivity Analysis of the Standard Deviation of the Co-polarized Phase Difference for Sea Oil Slick Observation. IEEE Trans. Geosci. Remote Sens. 2019, 57, 2022-2030. [CrossRef]

19. Skrunes, S.; Brekke, C.; Eltoft, T. Characterization of Marine Surface Slicks by Radarsat-2 Multipolarization Features. IEEE Trans. Geosci. Remote Sens. 2014, 52, 5302-5319. [CrossRef]

20. Li, H.; Perrie, W.; He, Y.; Wu, J.; Luo, X. Analysis of the Polarimetric SAR Scattering Properties of Oil-Covered Waters. IEEE J. Selected Topics Appl. Earth Obs. Remote Sens. 2015, 8, 3751-3759. [CrossRef]

21. Yin, J.; Yang, J.; Zhou, Z.-S.; Song, J. The Extended Bragg Scattering Model-Based Method for Ship and Oil-Spill Observation Using Compact Polarimetric SAR. IEEE J. Selected Topics Appl. Earth Obs. Remote Sens. 2015, 8, 3760-3772. [CrossRef]

22. Clemente-Colón, P.; Yan, X.-H. Low-Backscatter Ocean Features in Synthetic Aperture Radar Imagery. Johns Hopkins APL Tech. Digest 2000, 21, 116-121.

23. Meng, H.; Wang, X.; Chong, J.; Wei, X.; Kong, W. Doppler Spectrum-Based NRCS Estimation Method for Low-Scattering Areas in Ocean SAR Images. Remote Sens. 2017, 9, 219. [CrossRef]

24. Raney, R.K. Theory and Measure of Certain Image Norms in SAR. IEEE Trans. Geosci. Remote Sens. 1985, 23, 343-348. [CrossRef]

25. Nunziata, F.; Migliaccio, M. On the COSMO-SkyMed PingPong Mode to Observe Metallic Targets at Sea. IEEE J. Ocean. Eng. 2013, 38, 71-79. [CrossRef]

26. Nunziata, F.; Migliaccio, M.; Li, X.; Ding, X. Coastline Extraction Using Dual-Polarimetric COSMO-SkyMed PingPong Mode SAR Data. IEEE Geosci. Remote Sens. Lett. 2014, 11, 104-108. [CrossRef]

27. Elfouhaily, T.; Guérin, C.A. A Critical Survey of Approximate Scattering Wave Theories From Random Rough Surfaces. Waves Random Med. 2004, 14, R1-R40. [CrossRef]

28. Nunziata, F.; Sobieski, P.; Migliaccio, M. The Two-Scale BPM Scattering Model for Sea Biogenic Slicks Contrast. IEEE Trans. Geosci. Remote Sens. 2009, 47, 1949-1956. [CrossRef]

29. Lemaire, D.; Sobieski, P.; Craeye, C.; Guissard, A. Two-scale models for rough surface scattering: Comparison between the boundary perturbation method and the integral equation method. Radio Sci. 2002, 37, 1001. [CrossRef]

30. Gade, M.; Alpers, W.; Hühnerfuss, H.; Wismann, V.R.; Lange, A. On the Reduction of the Radar Backscatter by Oceanic Surface Films: Scatterometer Measurements and Their Theoretical Interpretation. Remote Sens. Environ 1998, 66, 52-70. [CrossRef]

31. Boukabara, S.A.; Eymard, L.; Guillou, C.; Lemaire, D.; Sobieski, P.; Guissard, A. Development of a modified two-scale electromagnetic model simulating both active and passive microwave measurements: Comparison to data remotely sensed over the ocean. Radio Sci. 2002, 37, 16-1-16-11. [CrossRef]

32. Guissard, A.; Sobieski, P.; Baufays, C. A unified approach to bistatic scattering for active and passive remote sensing of rough ocean surfaces. Trends Geophys. Res. 1992, 1, 43-68.

33. Grieco, G.; Lin, W.; Migliaccio, M.; Nirchio, F.; Portabella, M. Dependency of the Sentinel-1 Azimuth Wavelength Cut-off on Significant Wave Height and Wind Speed. Int. J. Remote Sens. 2016, 37, 5086-5104. [CrossRef]

34. Lombardini, P.; Fiscella, B.; Trivero, P.; Cappa, C.; Garrett, W. Modulation of the Spectra of Short Gravity Waves by Sea Surface Films: Slick Detection and Characterization With a Microwave Probe. J. Atmos. Ocean. Technol. 1989, 6, 882-890. [CrossRef]

35. Hasselmann, K.; Hasselmann, S. On the Nonlinear Mapping of an Ocean Wave Spectrum into a Synthetic Aperture Radar Image Spectrum and Its Inversion. J. Geophys. Res. Oceans 1991, 96, 10713-10729. [CrossRef]

36. Vachon, P.W.; Raney, R.K. Ocean Waves and Optimal SAR Processing: Don't Adjust the Focus! IEEE Trans. Geosci. Remote Sens. 1992, 30, 627-629. [CrossRef]

37. Vachon, P.W.; Krogstad, H.E.; Paterson, J.S. Airborne and Spaceborne Synthetic Aperture Radar Observations of Ocean Waves. Atmos. Ocean 1994, 32, 83-112. [CrossRef]

38. Kerbaol, V.; Chapron, B.; Vachon, P.W. Analysis of ERS-1/2 Synthetic Aperture Radar Wave Mode Imagettes. J. Geophys. Res. 1998, 103, 7833-7846. [CrossRef]

39. Corcione, V.; Grieco, G.; Portabella, M.; Nunziata, F.; Migliaccio, M. A Novel Azimuth Cutoff Implementation to Retrieve Sea Surface Wind Speed From SAR Imagery. IEEE Trans. Geosci. Remote Sens. 2019, 57, 3331-3340. [CrossRef]

40. Mera, D.; Cotos, J.M.; Varela-Pet, J.; Garcia-Pineda, O. Adaptive Thresholding Algorithm Based on SAR Images and Wind Data to Segment Oil Spills Along the Northwest Coast of the Iberian Peninsula. Marine Poll. Bullet. 2012, 64, 2090-2069. [CrossRef] [PubMed]

41. Amos, J. Satellite Imagery Reveals Scope of Last Week's Oil Spill in Kuwait. Skytruth Report. 2017. Available online: https: / / skytruth.org/2017/08/satellite-imagery-reveals-scope-of-last-weeks-massive-oil-spill-in-kuwait/ (accessed on 11 March 2020).

42. ESI. Major Oil Spill in the Mediterranean. European Space Imaging Press. 2018. Available online: https://www.euspaceimaging com/major-oil-spill-in-the-mediterranean/ (accessed on 11 March 2020).

43. ESA. Applications: Mediterranean Slick. European Space Agency Press. 2018. Available online: https://www.esa.int/ESA_ Multimedia/Images/2018/10/Mediterranean_slick (accessed on 11 March 2020).

44. Thomas, C. Oil Spill in the Persian Gulf. Shytruth Report; 2017. Available online: https://skytruth.org/2017/03/oil-spill-in-thepersian-gulf/ (accessed on 15 July 2019). 
45. ESA. Applications: Earth From Space-Gotland Baltic Blooms. European Space Agency Press. 2019. Available online: http: //www.esa.int/ESA_Multimedia/Videos/2019/12/Earth_from_Space_Gotland_Baltic_blo- \oms (accessed on 11 March 2020).

46. Koto, J.; Putrawidjaja, M. Subsea Pipeline Damaged in Balikpapan Bay Caused by Anchor Load. J. Subsea Offshore Sci. Eng. 2018, 14, 6-12.

47. Setiani, P.; Ramdani, F. Oil Spill Mapping Using Multi-sensor Sentinel Data in Balikpapan Bay, Indonesia. In Proceedings of the 2018 4th IEEE International Symposium on Geoinformatics (ISyG), Malang, Indonesia, 17-18 November 2018; pp. 1-4.

48. Gouveia, N.D.A.; Alves, F.C.; Pereira, L.D.O. Pre-processing of Sentinel-1 C-Band SAR Images Based on Incidence Angle Correction for Dark Target Detection. Remote Sens. Lett. 2019, 10, 939-948. [CrossRef]

49. ESA. Sentinel-1 Product Specification. Sentinel-1 User Handbook, 2016. Available online: https://sentinels.copernicus.eu/ documents/247904/685163/Sentinel-1_User_Handbook (accessed on 10 September 2020).

50. Nunziata, F.; de Macedo, C.R.; Buono, A.; Velotto, D.; Migliaccio, M. On the Analysis of a Time Series of X-band TerraSAR-X SAR Imagery Over Oil Seepages. Int. J. Remote Sens. 2019, 40, 3623-3646. [CrossRef]

51. Topouzelis, K.; Singha, S.; Kitsiou, D. Incidence angle Normalization of Wide Swath SAR Data for Oceanographic Applications. Open Geosci. 2016, 8, 450-464. [CrossRef] 